\title{
Direct Observation of Magnetic Interactions at Structural and Compositional Interfaces in Fe-Ti Oxides Using Lorentz Microscopy and Electron Holography
}

\author{
T. Kasama, ${ }^{*}$ R.J. Harrison, ${ }^{* *}$ N.S. Church, ${ }^{* *}$ J.M. Feinberg, ${ }^{* * *}$ and R.E. Dunin-Borkowski* \\ * Center for Electron Nanoscopy, Technical University of Denmark, DK-2800 Lyngby, Denmark \\ ** Department of Earth Sciences, University of Cambridge, Downing Street, Cambridge CB2 3EQ, \\ United Kingdom \\ *** Department of Geology and Geophysics, University of Minnesota, 310 Pillsbury Drive S.E., \\ Minneapolis, MN 55455-0219.
}

Local variations in the microstructure and chemical composition of combinations of magnetite $\left(\mathrm{Fe}_{3} \mathrm{O}_{4}\right)$, ulvöspinel $\left(\mathrm{Fe}_{2} \mathrm{TiO}_{4}\right)$, hematite $\left(\mathrm{Fe}_{2} \mathrm{O}_{3}\right)$ and ilmenite $\left(\mathrm{FeTiO}_{3}\right)$, which are of central importance in rock magnetism and of interest for the development of future spintronic devices, have a profound influence on their magnetic properties. Here, we illustrate how high spatial resolution magnetic information about Fe-Ti oxides can be obtained by using the techniques of Lorentz imaging and off-axis electron holography in the transmission electron microscope (TEM).

Figure 1 shows Lorentz TEM (out-of-focus) images of a magnetite specimen acquired using a Philips CM300 field emission gun TEM operated at $300 \mathrm{kV}$. The images were recorded below the Verwey transition, at which the magnetocrystalline anisotropy increases by an order of magnitude and the magnetic easy axis switches from the $<111>$ directions of the parent cubic phase to the [001] direction of the low temperature monoclinic phase. On cooling through the transition, the easy axis of the monoclinic phase can lie along any of the three $<100>$ directions of the cubic phase, resulting in the formation of transformation twins. At low temperature we observe micrometer-scale monoclinic twin domains (referred to here as Type 1 twins) that are separated from each other by jagged twin walls. The twin domains are subdivided further by lamellar twins (referred to here as Type 2 twins), which often join at their ends to form needle twins that move in response to thermal or magnetoelastic stress at temperatures below the Verwey transition by the advancement and retraction of their needle tips. Lateral motion of Type 1 and Type 2 twin walls is not generally observed, other than within a few degrees of the phase transition temperature. Figures 1 (c) - (f) show a $180^{\circ}$ magnetic domain wall being driven to the right by an increasing applied magnetic field, resulting in the conversion of a conventional $90^{\circ}$ domain wall into a 'divergent' $90^{\circ}$ domain wall [1].

Figure 2 shows magnetic induction maps recorded at room temperature using off-axis electron holography in an FEI Titan field emission gun TEM at $300 \mathrm{kV}$ from a sample that contains two different magnetic phases with measured compositions of (60\% hematite - $40 \%$ ilmenite) and (90\% magnetite - $10 \%$ ulvospinel). The hematite phase is a spin-canted antiferromagnet at room temperature, whereas the magnetite phase is a ferrimagnet. The images, which were recorded with the sample in magnetic-field-free conditions after applying different magnetic fields to the specimen, show the presence of magnetic vortex and anti-vortex states and illustrate the complexity of the magnetic coupling between the antiferromagnetic hematite and ferrimagnetic magnetite phases [2].

\section{References}

[1] T. Kasama et al., Earth and Planetary Science Letters 297 (2010) 10.

[2] R.J. Harrison, Reviews in Mineralogy and Geochemistry 39 (2000) 175. 


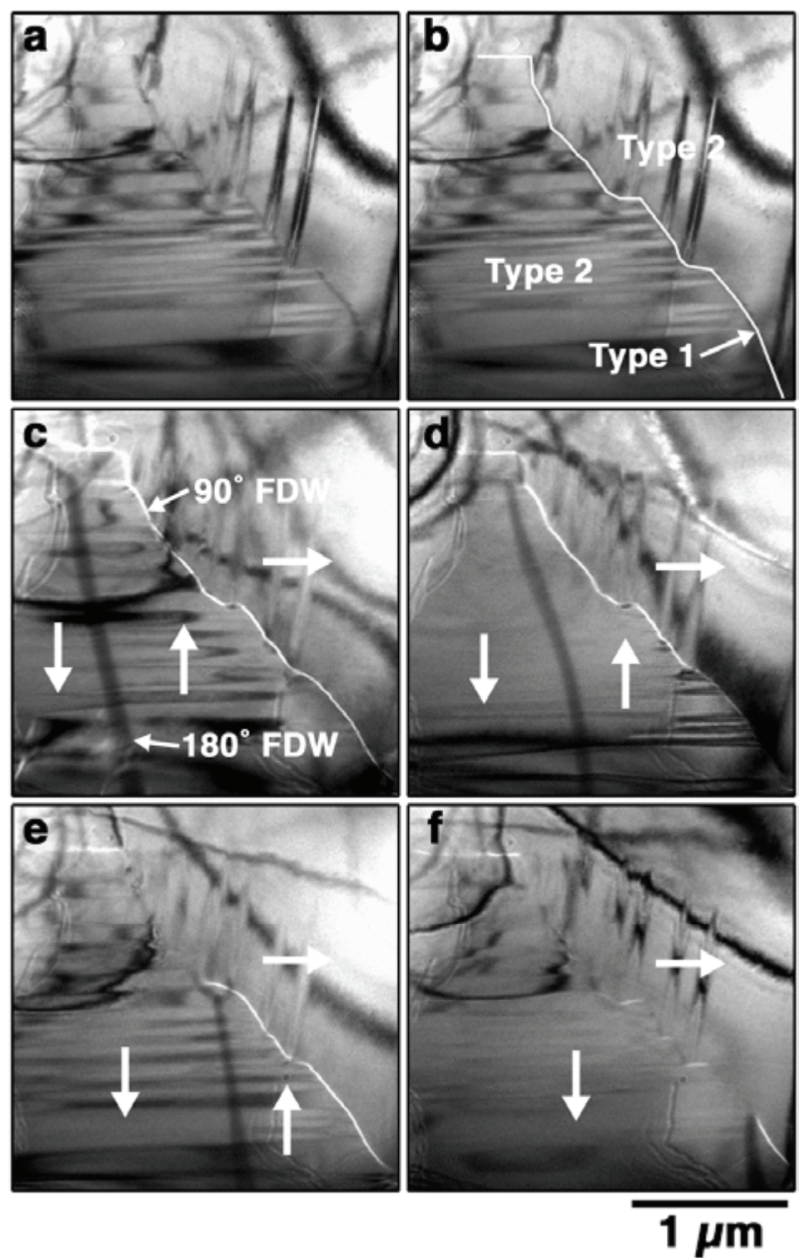

FIG. 1. (a, b) Bright-field TEM images of a magnetite specimen that contains 'Type 1' and 'Type 2' ferroelastic twins. The images were recorded close to focus with the sample cooled below the Verwey transition. Diffraction contrast in differently oriented twins and curving bend contours are visible. A Type 1 twin boundary is marked with a white line in (b). The region to the left of this boundary has its [001] magnetic easy axis oriented top-to-bottom. The region to the right has its easy axis oriented left-to-right. (c-f) Lorentz TEM images recorded from the same region in different (downward) fields showing ferrimagnetic domain walls (FDWs). The magnetic fields were applied by tilting the specimen in a 920 Oe vertical field generated by partially exciting the TEM objective lens. The in-plane components of the applied field are: (c) 20, (d) 50, (e) 80 and (f) 150 Oe.

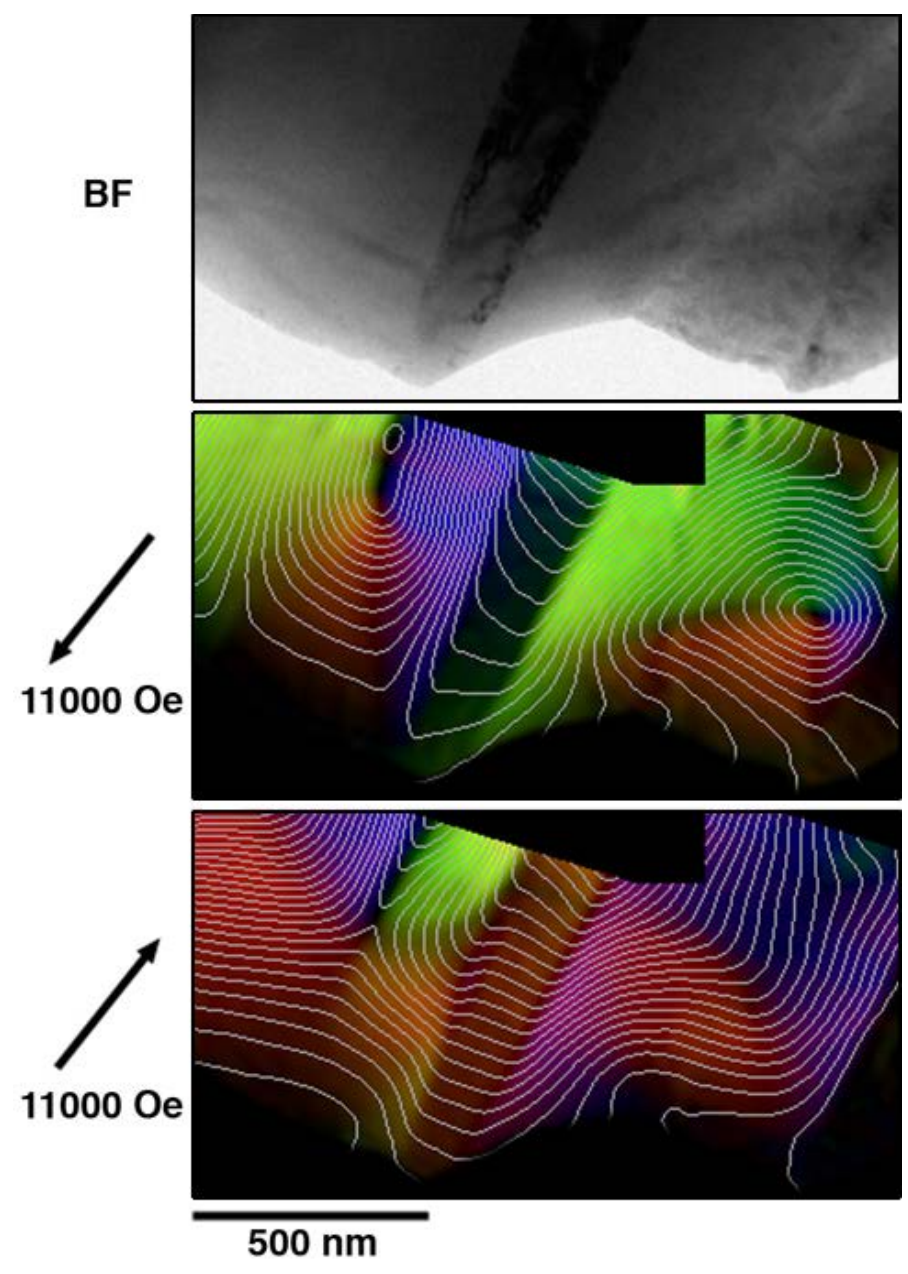

FIG. 2. Bright-field TEM image (top) and magnetic induction maps (bottom) recorded at room temperature in magnetic-field-free conditions using off-axis electron holography. The sample contains regions of a ferrimagnetic Ti-containing magnetite phase $(90 \%$ magnetite $10 \%$ ulvospinel) and regions of a spin-canted antiferromagnetic Ti-containing hematite phase (60\% hematite - 40\% ilmenite). The arrows show the direction and magnitude of the inplane component of the magnetic field applied to the specimen before recording electron holograms with the sample at remanence. The hematite phase appears darker as it does not have a significant moment at room temperature [2]. In the lowest image an antivortex-like magnetic state is visible. The complexity of the maps suggests that magnetic coupling at interfaces between the two phases influences their magnetic states strongly. 